Annales Geophysicae (2002) 20: 1167-1179 (C) European Geophysical Society 2002

\title{
Imaging radar observations and nonlocal theory of large-scale plasma waves in the equatorial electrojet
}

\author{
D. L. Hysell ${ }^{1,2,3}$ and J. L. Chau ${ }^{4}$ \\ ${ }^{1}$ Department of Physics and Astronomy, Clemson University, Clemson, South Carolina, USA \\ ${ }^{2}$ On leave at the Radio Science Center for Space and Atmosphere, Kyoto University, Uji, Kyoto 611-011, Japan \\ ${ }^{3}$ Now at the Department of Earth and Atmospheric Sciences, Cornell University, Ithaca, New York, USA \\ ${ }^{4}$ Radio Observatorio de Jicamarca, Instituto Geofísico del Perú, Lima, Peru
}

Received: 6 September 2001 - Revised: 25 January 2002 - Accepted: 6 February 2002

\begin{abstract}
Large-scale $(\lambda \sim 1 \mathrm{~km})$ waves in the daytime and nighttime equatorial electrojet are studied using coherent scatter radar data from Jicamarca. Images of plasma irregularities within the main beam of the radar are formed using interferometry with multiple baselines. These images are analyzed according to nonlocal gradient drift instability theory and are also compared to nonlinear computer simulations carried out recently by Ronchi et al. (1991) and Hu and Bhattacharjee (1999). In the daytime, the large-scale waves assume a non-steady dynamical equilibrium state characterized by the straining and destruction of the waves by shear and diffusion followed by spontaneous regeneration as predicted by Ronchi et al. (1991). At night, when steep plasma density gradients emerge, slowly propagating large-scale vertically extended waves predominate. Eikonal analysis suggests that these waves are trapped (absolutely unstable) or are nearly trapped (convectively unstable) and are able to tunnel between altitude regions which are locally unstable. Intermediate-scale waves are mainly transient (convectively stable) but can become absolutely unstable in narrow altitude bands determined by the background density profile. These characteristics are mainly consistent with the simulations presented by $\mathrm{Hu}$ and Bhattacharjee (1999). A new class of large-scale primary waves is found to occur along bands that sweep westward and downward from high altitudes through the E-region at twilight.
\end{abstract}

Key words. Ionosphere (equatorial ionosphere; ionospheric irregularities; plasma waves and instabilities)

\section{Introduction}

Large-scale waves were first detected in the equatorial electrojet two decades ago by radar interferometry and are now believed to dominate the structure and dynamics of the region. These waves are produced by the gradient drift instabil-

Correspondence to: D. L. Hysell

(daveh@geology.cornell.edu) ity throughout the day and night, except briefly near sunrise and sunset when the ionospheric electric field reverses. They propagate horizontally in the direction opposite the electrojet current and have wavelengths comparable to or longer than the background plasma density gradient scale length. Their intricate structure is hinted at by radar Doppler spectrograms, interferograms, and high-resolution range time intensity (RTI) backscatter maps (Kudeki et al., 1982; Swartz and Farley, 1994). The waves also dominate density and electric field irregularities observed by sounding rockets (Pfaff et al., 1982, 1987; Pfaff, 1991). In recent years, large-scale waves have undergone numerous theoretical and numerical investigations (see Farley, 1985; Kudeki et al., 1985; Ronchi, 1990; $\mathrm{Hu}$ and Bhattacharjee, 1998, for reviews). The theory of daytime waves in particular, has matured to the point of driving the development of new experimental techniques to validate it.

Large-scale waves in the nighttime electrojet, meanwhile, are poorly understood by comparison and are neither as well characterized nor as readily analyzed as the daytime waves. Sounding rockets are the only means of probing the electrojet in situ, but the majority of the rocket experiments devoted explicitly to electrojet studies were carried out in daytime. The few nighttime electrojet sounding rocket data sets available reveal electron density profiles that are jagged and irregular (e.g. Prakash et al., 1972; Pfaff et al., 1982). Neutral winds appear to substantially alter the polarization electric field in the electrojet, particularly around and after sunset (Kudeki et al., 1987; Reddy and Devasia, 1981; Hysell and Burcham, 2000; Hysell et al., 2002). The passage of the solar terminator introduces steep horizontal conductivity gradients into the electrojet region and induces a more complicated current system involving vertical currents that link directly with the F-region overhead (Haerendel et al., 1992; Haerendel and Eccles, 1992). Numerical models of nighttime large-scale waves need to incorporate these shears, currents, and conductivity gradients self consistently, in order to give a complete theoretical description of the nighttime case.

Much of what is known about large-scale electrojet waves 


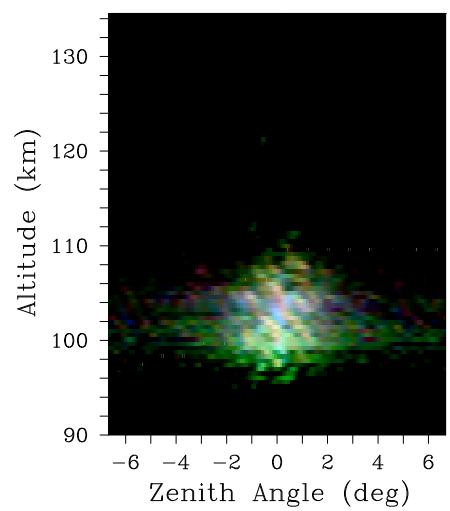

$18: 14: 09$

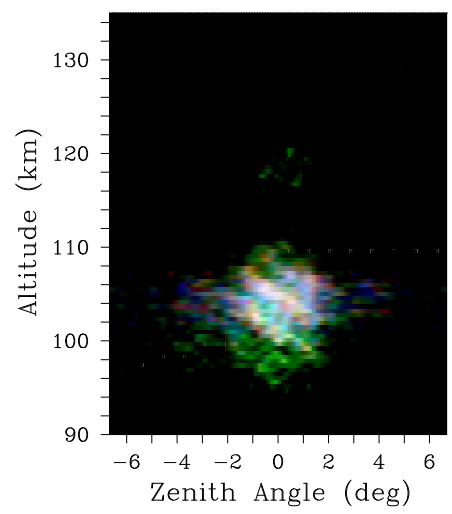

18:03:37

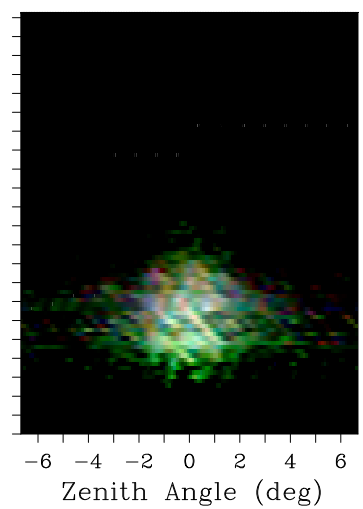

$18: 14: 25$

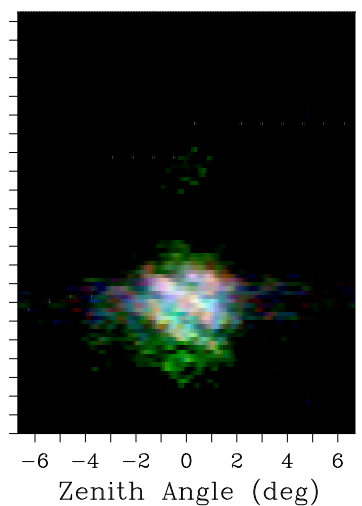

18:03:40

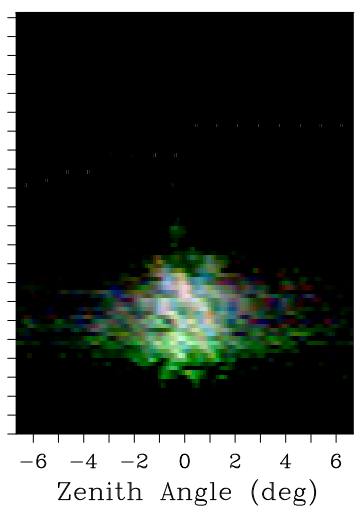

$18: 14: 41$

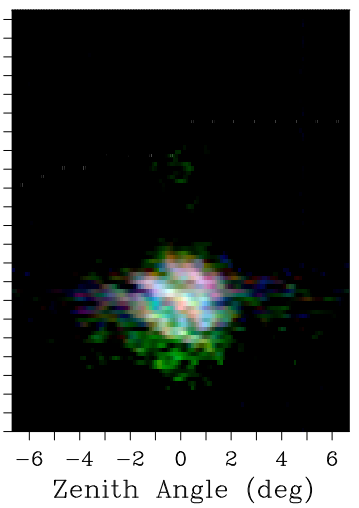

18:03:44

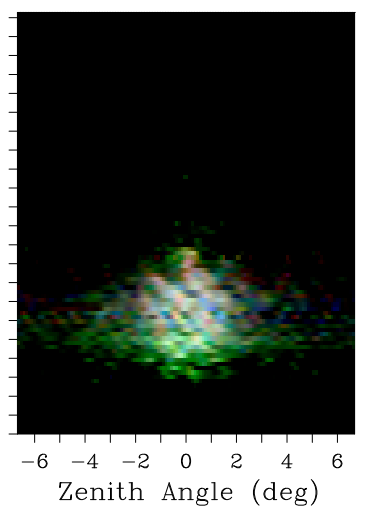

$18: 14: 56$

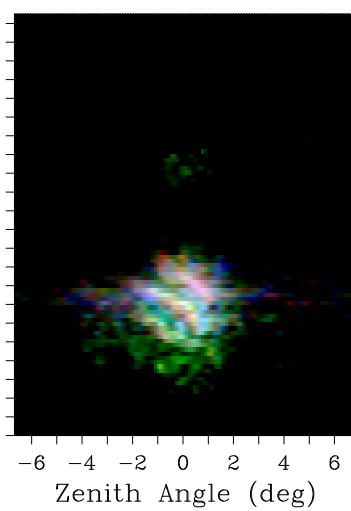

18:03:48

Fig. 1. Radar images of daytime electrojet waves. (Top row) 6 November 2000. (Bottom row) 8 November 2000. The lightness, hue, and saturation of each pixel in the images represent the backscatter intensity, Doppler shift, and spectral width of the echoes as a function of range in $\mathrm{km}$ and zenith angle in degrees.

was discovered using coherent scatter radars. However, measurements from conventional, monostatic, fixed-beam radars are inherently ambiguous; spatial and temporal variations in the scattering medium generally cannot be distinguished unless the velocity of the scatterers transverse to their line of sight is known. Moreover, the scattering volume of a radar, even as large as Jicamarca, is comparable in dimension to the wavelength of the waves in question here, making the waves difficult or impossible to resolve. Radar interferometry using a single baseline mitigates these problems and has led to the discovery of large-scale electrojet waves (Farley et al., 1981; Kudeki et al., 1982).

This paper presents observations of large-scale waves in the electrojet made with a new in-beam radar imaging technique. The technique, introduced at Jicamarca by Kudeki and Sürücü (1991) and developed by Woodman (1997) and Hysell and Woodman (1997), utilizes multiple interferometry baselines to resolve spatial-temporal ambiguity and to discern fine-scale structure within the radar scattering volume. We show high-resolution images of individual primary waves and wave packets propagating through the equatorial electrojet over Jicamarca within the radar beam. Animated sequences of images highlight the dispersion characteristics of the large-scale waves there and also give evidence of new wave phenomena in the electrojet which have not been previously detected. The images form the basis for direct model data comparisons and provide a more complete picture of the large-scale waves than was available previously.

This paper begins with a presentation of in-beam imaging observations of large-scale waves in the daytime and nighttime electrojet made at Jicamarca. Contemporary theories of large-scale waves are then reviewed. The theories provide jumping-off points for interpreting the main features of the images. We argue that the nonlinear theory of Ronchi et al. (1991) accurately describes the dynamical equilibrium state evident in the electrojet in the daytime. An extension of nonlocal, linear theory offers a qualitative explanation for the characteristics of the large-scale waves observed at night, which appear to be mainly consistent with the numerical simulations described by Hu and Bhattacharjee (1999).

\section{Imaging radar observations}

Images of the backscatter brightness distribution (the distribution of backscatter intensity versus arrival angle) within the beam of a radar can be formed from interferometry data taken with multiple baselines. It is well-known that interferometry with a single baseline yields the first and second 


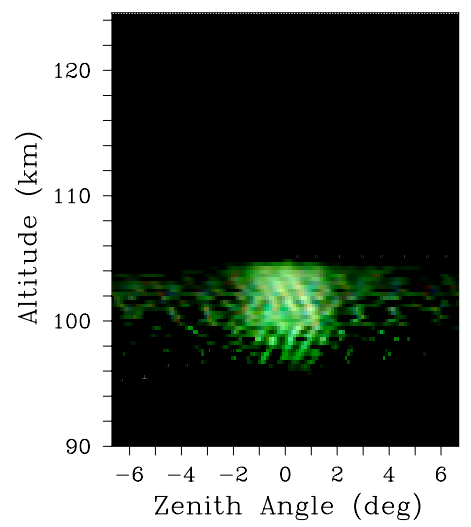

$15: 42: 16$

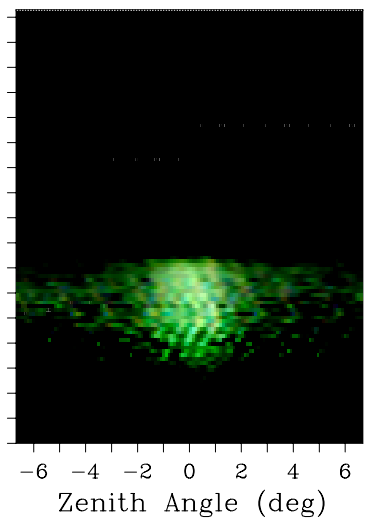

$15: 42: 23$

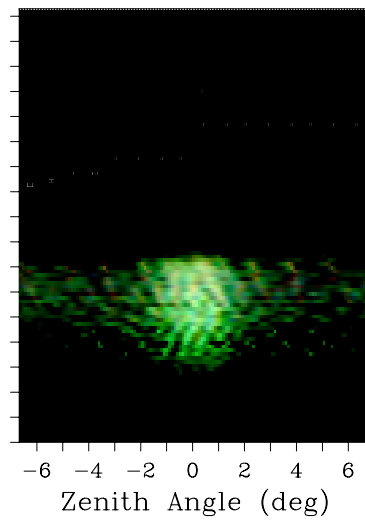

$15: 42: 31$

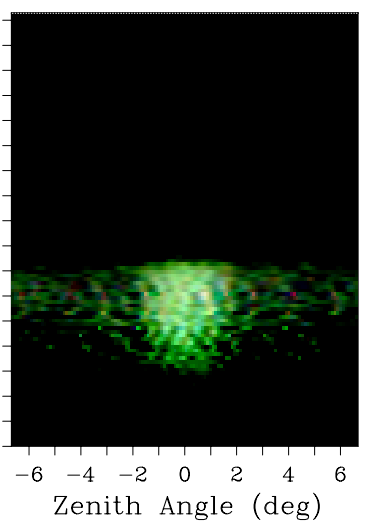

$15: 42: 39$

Fig. 2. Images of daytime large-scale waves observed on 7 November 2000 . No shear is evident in the flow here.

moments of the brightness distribution (Farley et al., 1981). More baselines yield more moments, and a sufficient number of moments define an image. Space-time ambiguities inherent in conventional radar work are resolved by the images which possess spatial degrees of freedom transverse to the radar beam. Furthermore, the spatial resolution of the technique is limited by the length of the longest baseline rather than the size of the main radar antenna array. The former can generally be increased readily, whereas the latter generally cannot, and it is possible in practice to form very high-resolution images even using radars with small main antenna arrays and broad beams. Images formed from different Doppler spectral components can be combined into composite images, highlighting the spectral characteristics of different regions of the scattering volume. Animated sequences of images depict how the scatterers evolve over time. All of the information in the scattered signal is fully utilized and objectively presented this way.

At Jicamarca, 6 nearly collinear antenna modules are used for reception. From them, the cross-correlations corresponding to 15 non-redundant interferometry baselines are computed. The longest of the baselines is 94 wavelengths. The measured cross-correlation or visibility function (crosscorrelation versus spatial antenna displacement) for each range gate is related to the desired brightness distribution by a Fourier transform (e.g. Thompson, 1986). Both functions can be regarded as one-dimensional since the scatterers in question are highly field-aligned. Since the visibility distribution is incompletely and imperfectly sampled in practice, and because the direct transformation from visibility to brightness space may be ill-conditioned, regularization techniques and statistical inverse theory are typically applied to the reconstruction of the brightness distribution from the data. The particular algorithm used here employs entropy as the regularization metric (Ables, 1974; Jaynes, 1982; Skilling and Bryan, 1984). A detailed description of the algorithm was given by Hysell and Woodman (1997).

Figure 1 shows in-beam radar images of coherent scatter from plasma irregularities in the daytime electrojet over Jica- marca. The vertical and horizontal axes of each image panel represent altitude in kilometers and zenith angle in the equatorial plane in degrees, respectively. Each pixel in the images is $0.1^{\circ}$ wide by 300 to $450 \mathrm{~m}$ high, and the incoherent integration time for each image is $4 \mathrm{~s}$. At an altitude of $100 \mathrm{~km}$, the zonal extent of the images is approximately $23 \mathrm{~km}$. The lightness of each pixel represents the signal-to-noise ratio on a $\log$ scale, typically from 3-30 dB. Since Jicamarca operates at a frequency close to $50 \mathrm{MHz}$, the lightness is an indication of the intensity of $3 \mathrm{~m}$ irregularities, which then act as tracers of intermediate- and large-scale waves. Note that the transmitting antenna mainly illuminates the central portion of the imaging field-of-view, explaining why the image peripheries are primarily dark. The hue of each pixel represents the first moment Doppler velocity, ranging typically from $-600 \mathrm{~m} / \mathrm{s}$ for red to $+600 \mathrm{~m} / \mathrm{s}$ for blue. The color scale is continuous, with green corresponding to zero Doppler shift. Finally, the saturation of each pixel represents the Doppler spectral width; pure (pastel) colors indicate narrow (broad) spectra.

The panels on the top row of Fig. 1, computed from data taken on 6 November 2000, are typical for images of the daytime electrojet. In them, alternating pink and blue bands can be seen propagating westward (toward the left). These are the alternating phases of primary gradient drift waves with wavelengths of a few $\mathrm{km}$. The bands are most distinct between about 97 and $109 \mathrm{~km}$ in altitude. The spectra underlying the echoes are broad type 2. Animated images reveal strong vertical shear in the apparent flow, which is $150 \mathrm{~m} / \mathrm{s}$ westward at $110 \mathrm{~km}$ altitude, stationary at $100 \mathrm{~km}$, and slow and eastward below $100 \mathrm{~km}$. Shear in the electrojet arises from altitude variations both in the electrojet current and the zonal neutral wind (Hysell et al., 2002). The waveforms strain under the shear, then twist and decrease in wavelength, and finally flatten over time. Eventually, they vanish even as new waveforms are generated. The lifetime of individual waveforms is on the order of tens of seconds to a minute. A sheared, non-steady dynamical equilibrium flow appears to be sustained. Similar remarks hold for the images in the bottom row of Fig. 1, which correspond to 8 November 2000. 


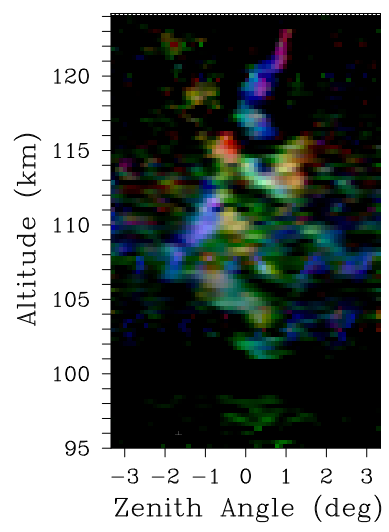

22:23:06

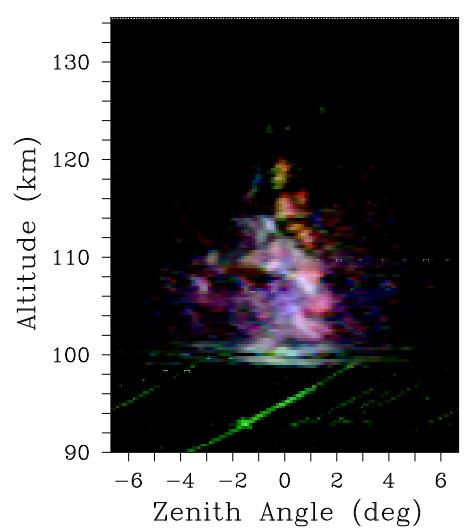

$21: 56: 26$

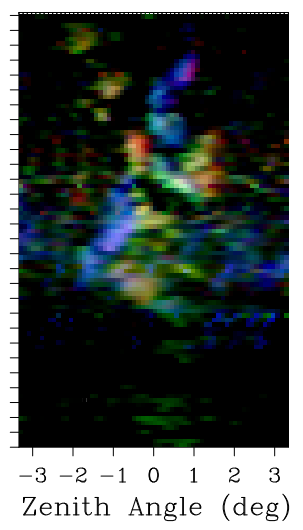

22:23:10

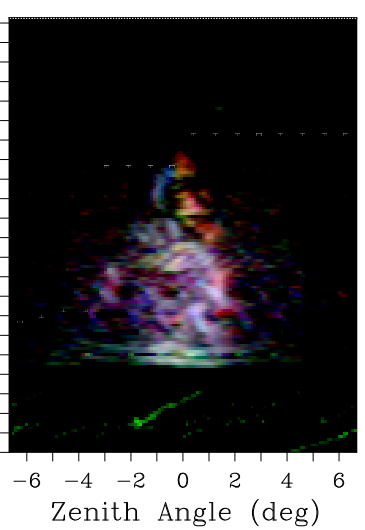

$21: 56: 34$

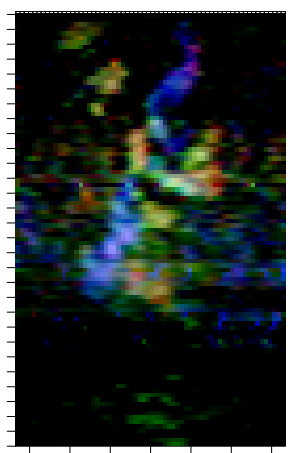

$\begin{array}{llllll}-3 & -2 & -1 & 0 & 1 & 2\end{array}$

Zenith Angle (deg)

$22: 23: 14$

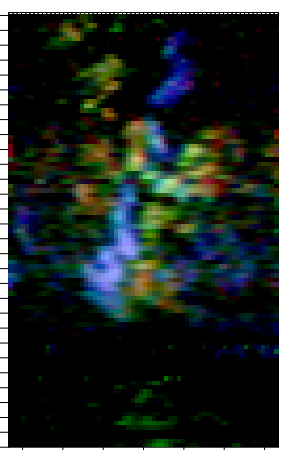

$\begin{array}{lllllll}-3 & -2 & -1 & 0 & 1 & 2 & 3\end{array}$

Zenith Angle (deg)

$22: 23: 18$

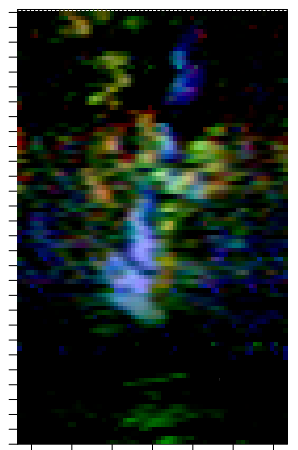

$\begin{array}{lllllll}-3 & -2 & -1 & 0 & 1 & 2 & 3\end{array}$

Zenith Angle (deg)

22:23:22

Fig. 3. Radar images of nighttime electrojet waves observed on 24 April 2000. Note the range-spread meteor evident in the images on the bottom row of the figure.

This time, however, the horizontal wavelength of the waves is about $3 \mathrm{~km}$.

The image panels in Fig. 2, which correspond to echoes observed a few hours earlier in the day on 7 November 2000, depict a qualitatively different situation. Regular waveforms with a wavelength of about $1 \mathrm{~km}$ are clearly evident in the images. The top of the scattering region is more sharply defined than the bottom, as might be expected on the basis of linear, local instability theory. This is due to the fact that the vertical plasma density gradient reverses sign in the E-region topside, an effect which is strongly stabilizing. Animation of the panels shows that the flow has relatively little shear this time. The westward propagation speed of the waves is only about $20 \mathrm{~m} / \mathrm{s}$. The waveforms visible in the images have long lifetimes, on the order of a few minutes.

Turning to the case of the nighttime electrojet, Fig. 3 shows images of large-scale electrojet irregularities observed on 24 April 2000 after the evening reversal of the zonal electric field. Note that the zenith angle span of the images on the top row is half that of all the other images. In the top row, we see the different phases of a tilted large-scale wave propagating eastward and downward through the scattering volume. In animations, one sees that all of the blue regions that appear to fall along the line from the top center to the lower left corner of the images move together as part of a wavefront. This is one phase of the wave. Opposing phases of the wave, which appear as disconnected lines of red and green regions in the images, both precede and trail the blueshifted phase. Many more phases follow continuously thereafter (not shown). However, the large-scale waves shown here behave more like wave packets than like a plane wave. The wavefronts evolve in time and change orientation as they propagate. Moreover, the amplitude of the backscatter is modulated in altitude, and the pattern of the amplitude modulation shifts in altitude on a time scale shorter than the period of the waves. The effect suggests that the wave packets subtend regions of space that are alternately stable and unstable. The eastward phase velocity of the wave seen here was never greater than about $50 \mathrm{~m} / \mathrm{s}$ and varied drastically with time and altitude. The wave packet extended over a $20 \mathrm{~km}$ altitude range and had a lifetime greater than its transit time through the radar beam. Different phases of the wave propagate alternatingly upward and downward; in RTI diagrams, the effect is to produce tightly interleaved, crossed diagonal lines (Swartz and Farley, 1994; Farley et al., 1994). Unlike the daytime case, these large-scale waves do not appear to strain and dissipate under the action of shear flow. Also, the wavelength of the waves increases at night, although it 


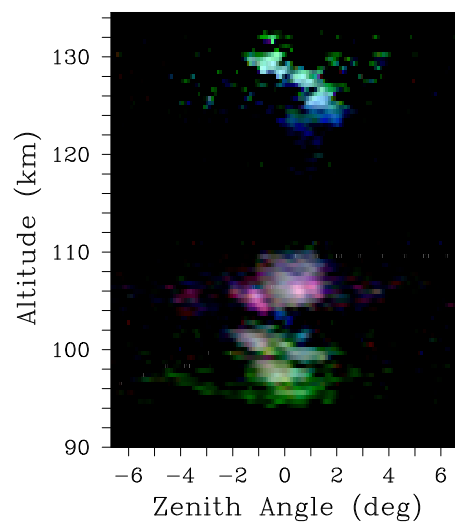

$18: 35: 52$

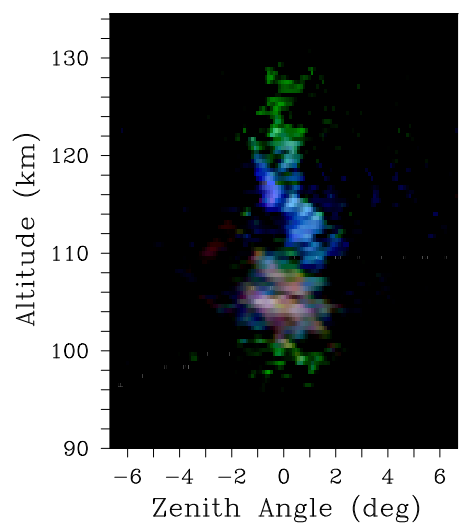

$18: 26: 35$

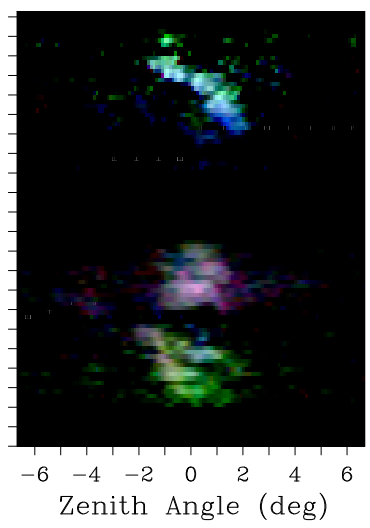

18:36:00

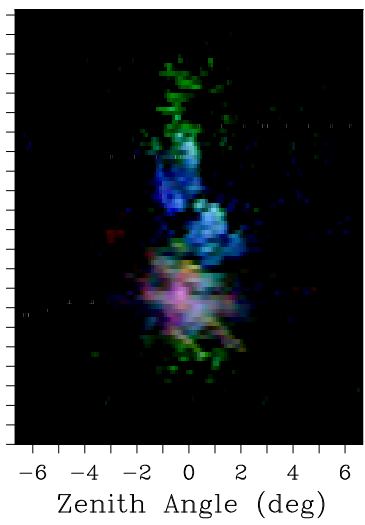

$18: 26: 43$

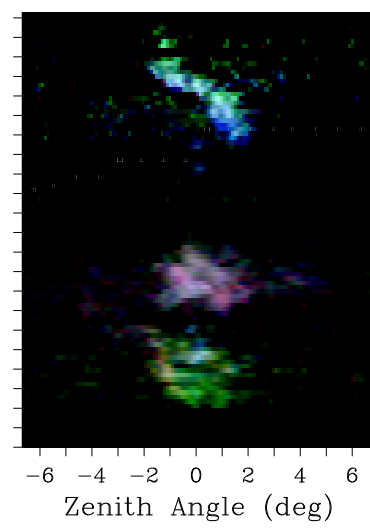

18:36:09

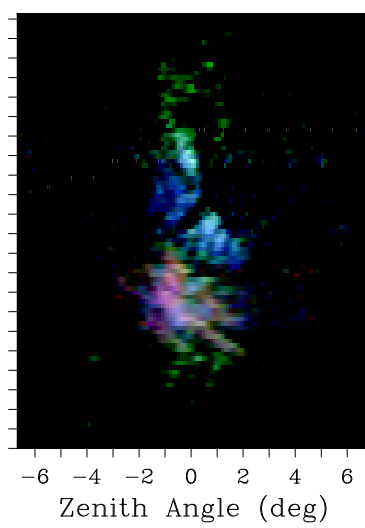

$18: 26: 51$

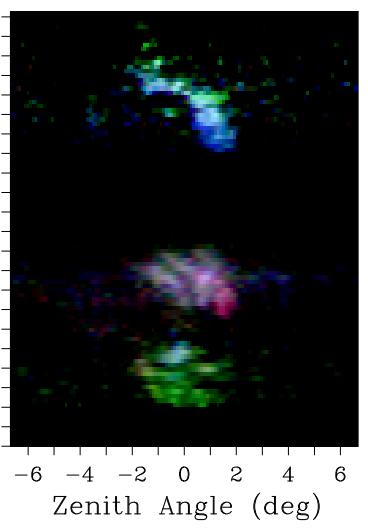

$18: 36: 1^{r}$

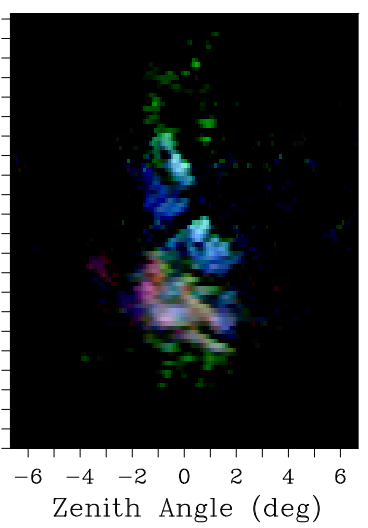

$18: 26: 59$

Fig. 4. Radar images corresponding to twilight conditions. (Top row) 24 April 2000. (Bottom row) 6 November 2000.

is difficult to estimate the wavelength since they are not so monochromatic as in the daytime.

Note that the blue-shifted wavefront in these images has a nearly pure blue color. Conventional spectra analysis shows that the echoes coincident in altitude with the packet have type 1 spectra. Clearly secondary Farley-Buneman instabilities are excited by the polarization electric field associated with the primary wave packet and the vertical Hall current it generates. This secondary wave generation mechanism was proposed by Sudan et al. (1973) and has been supported by numerous investigations since. Here, we see the mechanism illustrated vividly.

Similar remarks hold for the waveforms shown in the bottom row of Fig. 3. Here, up-going and down-going phases of the eastward-propagating waves are shown interleaved. In addition, there are bright, diffuse bands of backscatter just above and below $100 \mathrm{~km}$ altitude. A distinct gap lies between the bands. These bands originally formed at about $21: 10 \mathrm{LT}$ at an altitude of $120 \mathrm{~km}$ and then descended in a slow arc until about 22:30 LT when they reached an altitude of about $96 \mathrm{~km}$ and, soon after, vanished. The primary gradient drift waves that make up these scattering layers evidently have too small a scale to be resolved here. We can hypothesize that a very steep plasma density layer presented a stable gradient originally at $120 \mathrm{~km}$ altitude and unstable gradients above and below that. The layer migrated downward over time in a manner reminiscent of an intermediate descending layer, except at E-region altitudes (Mathews et al., 1997).

The signature of a range-spread meteor echoes is clearly visible in the first two frames of Fig. 3. Such trails are quite common in the data and often persist for tens of seconds. Coincidentally, this trail passed through a scattering blob at $94 \mathrm{~km}$ that appeared abruptly in the images at about 21:55 LT, at precisely the moment and location of another range spread meteor echo. The blob then drifted slowly westward and remained visible until about 21:57 LT. The first meteor seems to have played some role in initiating plasma instabilities in the lower E-region. Similar phenomena have been discussed by Chapin and Kudeki (1994) and have also been observed at mid-latitudes (Hysell and Burcham, 1999).

Finally, Fig. 4 presents an aspect of large-scale waves in the equatorial electrojet not previously recognized or predicted. The figure shows images taken around twilight on 24 April 2000 (top) and 6 November 2000 (bottom). On both days, lines of scatterers formed at an altitude of about $130 \mathrm{~km}$ and then swept downward and westward through the radar field-of-view. The velocity of the scatterers was about $50 \mathrm{~m} / \mathrm{s}$. The scattering lines were highly structured and seemed to exhibit the signatures of primary gradient drift waves with wavelengths on the order of $1 \mathrm{~km}$. The Doppler 
shifts of the echoes were positive and, in some places, approached the ion acoustic speed.

This phenomenon is evident in several other imaging data sets and is frequently detected by the JULIA (Jicamarca Unattended Long-term investigation of the Ionosphere and Atmosphere) radar at Jicamarca, where it appears as clusters of high-altitude E-region echoes that occur around 18:30 LT. Sometimes, several parallel lines of scatterers sweep through the scattering volume sequentially. While the nature of the echoes is not known, we tentatively associate them with a gradient drift or interchange instability driven by the horizontal conductivity gradients and vertical (upward) electrojet currents known to be present in the vicinity of the solar terminator. A detailed analysis of the phenomenon remains to be performed.

In the next section of the paper, we evaluate contemporary theories of large-scale waves in the equatorial electrojet in light of the radar images. Theoretical work in the case of gradient drift waves in the daytime is extensive but conflicting. Ronchi et al. (1991) argued that intermediate- and smallscale waves in the daytime are convectively stable in sheared flows, but that large-scale waves are continuously regenerated by nonlinear mode coupling, leading to their predominance. We will argue that the daytime radar images, which show evidence of a non-steady dynamical equilibrium state when shear is present, support this theory. In the absence of sheared flow, meanwhile, a linear, local theory which includes anomalous effects alone can account for the predominance of large-scale waves in the daytime. Existing theoretical work in the case of nighttime gradient drift waves is, by comparison to the daytime case, less mature. We will show with a linear, nonlocal analysis that, at night, the short gradient scale lengths associated with jagged density profiles cause large-scale waves to be absolutely or convectively unstable. These waves are highly dispersive and propagate very slowly. Trapped and nearly-trapped wave modes can tunnel between regions of space that are locally unstable and, in so doing, extend over a large altitude range. Such tunnelling, which was predicted in the computational studies of $\mathrm{Hu}$ and Bhattacharjee (1999) but which is an inherently linear phenomenon, is evidenced by the nighttime radar images. Intermediate-scale waves can also be absolutely unstable at night in narrow altitude bands where the plasma density gradient is locally favorable. These bands can sometimes be seen in the nighttime radar images and were also predicted by $\mathrm{Hu}$ and Bhattacharjee (1999).

\section{Theoretical background}

Large- and intermediate-scale plasma waves are produced in the equatorial electrojet directly by the gradient drift instability (Simon, 1963; Hoh, 1963). A linear, local dispersion relation for the waves can be derived by combining the linearized fluid equations for the electron and ion gases and incorporating appropriate equations of state. By assuming plane wave perturbations for the field quantities of the form $\exp (i(\mathbf{k} \cdot \mathbf{x}-\omega(\mathbf{k}) t))$, where $\mathbf{x}$ is the displacement in the plane perpendicular to the geomagnetic field and $\mathbf{k}$ the wave vector, the real and imaginary parts of the frequency $\omega=\omega_{r}+i \gamma$ of purely field-aligned waves can be determined (see, for example, Kudeki et al. (1982)):

$$
\begin{aligned}
\omega_{r} & =\frac{\mathbf{k} \cdot\left(\mathbf{v}_{d e}-\mathbf{v}_{d i}\right)}{(1+\psi)\left(1+k_{\circ}^{2} / k^{2}\right)}+\mathbf{k} \cdot \mathbf{v}_{d i}, \\
\gamma & =\frac{k_{\circ}}{k}\left(\omega_{r}-\mathbf{k} \cdot \mathbf{v}_{d i}\right)-k^{2} D_{\perp}-2 \alpha n_{\circ}, \\
\frac{k_{\circ}}{k} & \equiv \frac{1}{1+\psi} \frac{k_{\|}}{k^{2} L} \frac{v_{i}}{\Omega_{i}} .
\end{aligned}
$$

Here, $\mathbf{v}_{d e}$ and $\mathbf{v}_{d i}$ are the zero-order transverse electron and ion drift velocities, respectively. The former is associated mainly with the Hall drift of the electrons in the electrojet, and the latter is due mainly to neutral wind effects. Also, $\alpha$ is the dissociative recombination rate, and $\psi$ is the anisotropy factor defined by $\psi=v_{e} v_{i} / \Omega_{e} \Omega_{i}$. The transverse diffusion is defined by $D_{\perp}=\frac{\psi}{v_{i}} k^{2} C_{s}^{2} /(1+\psi)$. The $v_{j}$ terms refer to the ion-neutral collision frequencies for species $j$. $C_{S}$ is the ion acoustic speed, $L$ is the vertical density gradient scale length, $k_{\|}$is the component of the wave-vector in the direction of $\mathbf{v}_{d e}$, and the other terms have their usual meaning.

\subsection{Daytime case}

Kudeki et al. (1982) performed the first detailed experimental study of primary gradient drift waves in the electrojet using both spectral analysis and interferometry at Jicamarca. They observed dominant, large-scale waves propagating horizontally with wavelengths between about 2 and $6 \mathrm{~km}$. Secondary, vertically propagating two-stream waves were shown to be generated by the electron convection associated with the large-scale waves as hypothesized by Sudan et al. (1973). Secondary gradient drift waves were also observed and are now thought to be generated by nonlinear mode coupling and plasma turbulence (Sudan, 1983). Perhaps most remarkably, the large-scale waves were observed propagating at phase speeds of only about half the nominal electron drift velocity. Kudeki et al. (1982) noted that the large-scale waves belonged to the $|\gamma| \sim\left|\omega_{r}\right|$ or the $k \sim k_{\circ}$ regime. By retaining the quadratic term in the denominator of (1) and considering the low-frequency limit of the dispersion relation, they accounted for the slow propagation speeds of the waves. They also suggested that the dispersive nature of the large-scale waves inhibited their dissipation through mode coupling and contributed to their long lifetimes and coherence. Finally, they argued that the predominance of waves with the observed wavelengths was at least roughly predicted by (1) and (2) in which the recombination term would seem to impose a long-wavelength cutoff at precisely $k \approx k_{\circ}$, where $k_{\circ} L \approx 23$ for typical daytime parameters.

However, Huba and Lee (1983) showed that the growth rate predicted by the linear, local dispersion relation actually peaks in the vicinity of $k L \approx 100$. Thus, local theory favors the existence of intermediate-scale waves, whereas largescale waves are observed to predominate. They attributed the 
discrepancy between theory and observation to the inapplicability of local theory to the large-scale waves. An eigenmode analysis was performed which allowed for vertical shear in the electrojet Hall current. Shear in physical space causes convection in Fourier space (convection of energy to higher wave numbers) and causes otherwise unstable intermediatescale waves to be diffusively damped. The waves most affected are those with wavelengths comparable to or smaller than the scale size of the shear. Meanwhile recombination establishes the outer scale for wave growth. Shear was shown to stabilize the small- and intermediate-scale modes of the gradient drift instability and to increase the wavelength of the fastest growing eigenmodes to $k L \sim 20$ over a broad range of daytime conditions.

Shortly thereafter, Fu et al. (1986) reexamined the role of shear in E- and F-region plasma instabilities. Despite the fact that intermediate-scale eigenmodes are damped by shear, one-dimensional initial value computer simulations of the sheared system predicted a strong transient response at intermediate and small scales. Fu et al. (1986) pointed out that the sheared system is non-normal and that the linear eigenmodes are, therefore, not necessarily orthogonal or complete. They termed the transient response a "quasi-local" mode, inaccessible to eigenmode analysis. The predicted, strong transient response at intermediate scales contradicted radar evidence of large-scale waves, with $k \approx k_{\circ}$ dominating the daytime electrojet.

Ronchi et al. (1989) revisited the problem of large-scale daytime waves, performing nonlocal analyses which considered height dependent plasma density, Hall current, and electron and ion mobility profiles. They performed an eikonal or geometric optics analysis along the lines of Weinberg (1962) which yielded a more transparent interpretation of the transient response of Fu et al. (1986). The methodology, which involved a WKB expansion about the local dispersion relation and which yields information about both the steady-state and transient behavior of a system, will be important for our analysis and is outlined below.

The Appendix gives linearized model equations for largescale gradient drift waves propagating in regions of the electrojet where the background plasma density, electric field, mobilities, and diffusivities as well are allowed to vary with height. The model has been Fourier transformed in the horizontal direction and in time; it will be evaluated at one value of $k_{x}$ at a time and is consequently one-dimensional. Assume that solutions to the equations for particular $k_{x}$ have the form

$u(z, t)=u_{\circ}(z, t) e^{i S(z, t)}$,

where $S$ is the eikonal which controls rapid variations in phase along the ray path, and $u_{\circ}$ is a slowly varying amplitude function. We define the local vertical wave vector and frequency, respectively, by $k_{z}(z, t) \equiv \partial S / \partial z$ and $\omega(z, t) \equiv-\partial S / \partial t$, where the frequency is real and obeys the local dispersion relation $\omega=W\left(k_{z} ; x, t\right)$. It can then be shown that $W$ plays the role of the Hamiltonian and that the trajectories of the ray paths in the $k_{z}-z$ plane are ballistic (e.g. Landau and Lifshitz, 1997):

$$
\frac{d z}{d t}=\frac{\partial W}{\partial k_{z}}, \quad \frac{d k_{z}}{d t}=-\frac{\partial W}{\partial z}, \quad \frac{d W}{d t}=\frac{\partial W}{\partial t} .
$$

If there is no explicit time variation in the local dispersion relation, then the wave frequency $\omega$ is a constant of motion along the ray, and contours of constant frequency give the orbits of wave packets propagating through the medium. Ronchi et al. (1989) extended the eikonal analysis to the dissipative case in which the frequency has real and imaginary parts. To a good approximation, the trajectories of the ray paths continue to follow contours of the real part of the frequency. However, the amplitude of the wave packets can be modified as they move through regions of physical and Fourier space in which the local growth rate is positive or negative.

We evaluated the local frequency and growth rate in the $k_{z}-z$ plane according to (A1) for daytime conditions and plotted the results on the top row of Fig. 5. This figure, calculated for waves with $k_{x}=1 \times 10^{-3} \mathrm{~m}^{-1}$, essentially reproduces Fig. 7 of Ronchi et al. (1989). The constant frequency contours represent trajectories in $k_{z}-z$ space for wave packets originating from background noise. A wave packet originating at the point marked " 1 " in the upper left panel will propagate to the left along an $\omega=$ const contour, experience growth for a time, but ultimately decay after exiting the unstable region delineated by the $\gamma=0$ curve in the upper right panel. This is a convectively stable, transient wave. A packet originating at point "2", meanwhile, will follow a closed contour and become trapped. In this case, the closed contour lies wholly within the zone where $\gamma>0$. A wave travelling on it will be absolutely unstable and corresponds to an unstable eigenmode.

Ronchi et al. (1989) produced another figure similar to Fig. 5, except calculated for intermediate-scale waves with $k_{x}=7 \times 10^{-2} \mathrm{~m}^{-1}$. In that case, the lack of dispersion caused the ray trajectories to be nearly straight horizontal lines. There were no closed contours, and all the wave solutions, therefore, corresponded to transient modes. Ronchi et al. (1989) interpreted the quasi-local mode of Fu et al. (1986) in this way. Ronchi et al. (1989) also pointed out that modes on open contours can be effectively trapped and absolutely unstable if they exist on a ridge in the $k_{z}-z$ plane where $\nabla W$ vanishes. Seeking an explanation for why the absolutely unstable intermediate-scale waves are not observed in the daytime, Ronchi et al. (1990) followed St.-Maurice (1987) and considered the effects of small-scale gradient drift turbulence on electron transport. An effective turbulent Pedersen electron mobility was derived as

$$
\mu_{\perp e}^{*} \equiv \frac{1}{2} \frac{\psi}{1+\psi}\left(\frac{\mu_{H e}}{\mu_{\perp e}}\right)^{2}\left\langle\left|\frac{\delta n}{n}\right|^{2}\right\rangle \mu_{\perp e}
$$

in which $\mu_{H e}$ is the Hall mobility and where the density fluctuations are those due to small-scale $(\lambda<100 \mathrm{~m})$ turbulence. The correction to the electron mobility, which can be much 

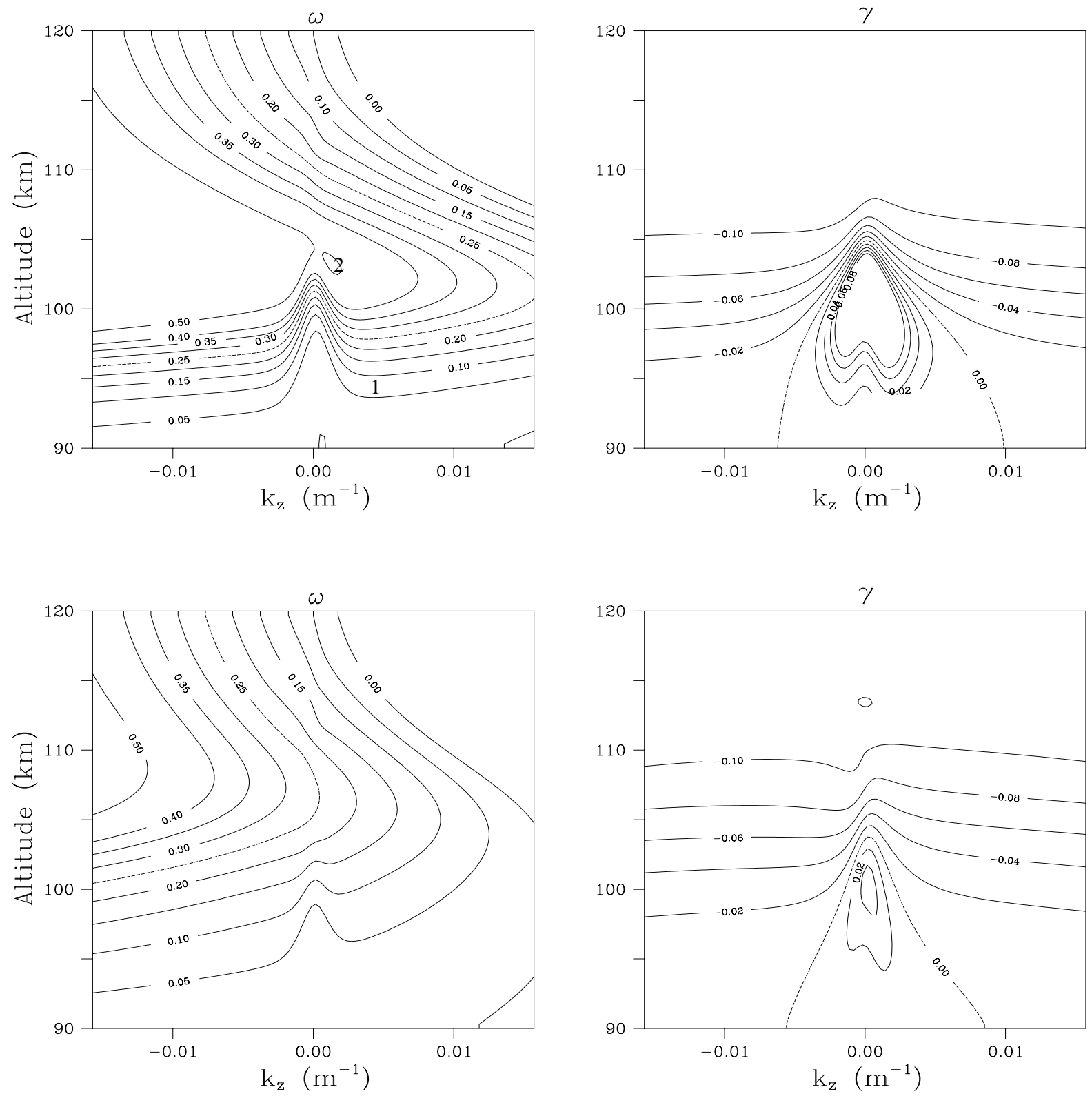

Fig. 5. Contours of constant frequency and growth rate derived from the local dispersion relation described in the Appendix for $k_{x}=1 \times 10^{-3} \mathrm{~m}^{-1}$, plotted in the $k_{z}-z$ plane. (Top) Daytime case with no anomalous electron mobility. (Bottom) Daytime case with anomalous electron mobility. The zonal electric field for both cases was taken to be $0.65 \mathrm{mV} / \mathrm{m}$. Model atmospheric and ionospheric parameters were derived from the International Reference Ionosphere (IRI) and Mass Spectrometer Incoherent Scatter (MSIS) models (Bilitza et al., 1993; Hedin et al., 1996). Collision frequencies were derived according to expression from Richmond (1972) and Gagnepain et al. (1977) and reproduced by Forbes (1981) in his Eqs. (27)-(30).

larger than the classical mobility given density fluctuations of a few percent, telegraphs through to the dispersion relation for gradient drift waves by increasing the electron Pedersen conductivity and, therefore, $\psi$. Furthermore, it alters the vertical polarization electric field profile in the electrojet, which is well approximated by the zonal electric field multiplied by the radio of the local Hall to Pedersen conductivities. The main effect here is to reduce significantly the vertical polarization electric field, particularly at altitudes below about
$105 \mathrm{~km}$. Ronchi et al. (1990) showed that anomalous electron mobility causes the fastest growing linear local gradient drift modes to occur at longer wavelengths and higher altitudes than would otherwise be predicted, independent of shear effects. By virtue of reducing the $k_{\circ} / k$ ratio for the fastest growing mode, it also causes the large-scale waves to be essentially non-dispersive. This implies that mode coupling between the waves can be very effective. Finally, the related reduction of the local vertical polarization electric field 

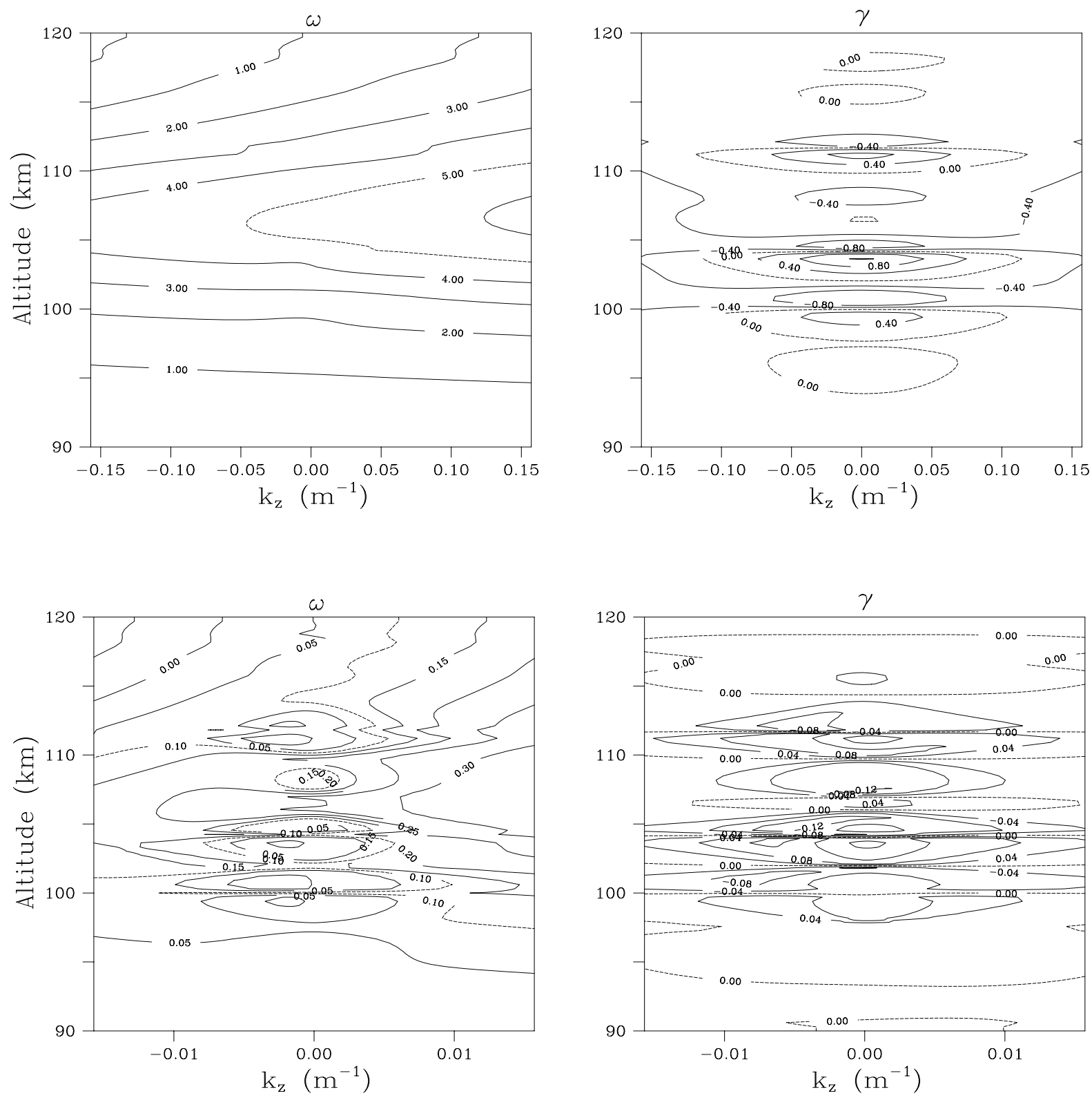

Fig. 6. Contours of constant frequency and growth rate derived from the local dispersion relation described in the Appendix and plotted in the $k_{z}-z$ plane. (Top) Nighttime case for waves with $100 \mathrm{~m}$ horizontal wavelengths. (Bottom) Nighttime case for waves with $2 \mathrm{~km}$ horizontal wavelengths. The zonal electric field for both cases was taken to be $-0.2 \mathrm{mV} / \mathrm{m}$. In both cases, a 0.04 RMS density fluctuation level was used to estimate the effects of anomalous electron mobility.

reduces the phase speeds of the waves to approximately $1 / 2$ to $2 / 3$ of the zonal electron drift speed, again independent of shear or low-frequency effects. These findings are important since the local dispersion relation ultimately determines which modes, transient and trapped, can arise.

The bottom panel of Fig. 5 shows dispersion curves calculated for waves with $k_{x}=1 \times 10^{-3} \mathrm{~m}^{-1}$, this time including the effects of small-scale turbulence with an RMS amplitude of 0.04. The curves indicate that the trapped modes have disappeared, leaving only transient waves. Likewise, Ronchi et al. (1990) showed that anomalous electron transport elim- inated the absolutely unstable intermediate-scale wave mode referred to previously. Consequently, all intermediate- and large-scale waves in the daytime electrojet were shown to be transient modes, convectively stable rather than absolutely unstable. Ultimately, Ronchi et al. (1991) would perform nonlinear simulations of the gradient drift instability to explain the persistence of the transient modes. Shear stabilizes all waves with wavelengths comparable to the shear gradient scale length by convection in Fourier space, which causes a shift from small wave numbers to large and leads to diffusive dissipation. However, the nonlinear mode coupling that 
takes place is such as to transport energy from large wave numbers back to small wave numbers. A dynamical equilibrium saturated state was predicted involving the shearing and reproduction of large-scale waves whose characteristics were predicted by the linear, local dispersion relation with anomalous effects included. Simulations preferentially produced transient waves with wavelengths of about $2 \mathrm{~km}$ and lifetimes of about $30 \mathrm{~s}$.

\subsection{Nighttime case}

The factors outlined above continue to influence the evolution of large-scale waves at night. Several new factors also emerge, however, including the drastic reduction of the dissociative recombination rate with the reduced plasma number density. This tends to relax the long-wavelength cutoff on the linear gradient drift growth rate and might be expected to increase the outer scale of the instability. This effect is balanced, however, by the emergence of layers and steep density gradients, as shown by Prakash et al. (1972) and others. Finite gradient scale length effects are expected to reduce the growth rate of large-scale waves and favor intermediatescale waves at night. By applying an initial value calculation to nighttime rocket data, Hu and Bhattacharjee (1999) predicted that the fastest growing linear nonlocal gradient drift modes should have wavelengths of only about $20 \mathrm{~m}$. This contradicts radar evidence that the wavelength of the dominant large-scale waves actually increases from day to night, however. Hu and Bhattacharjee (1999) ultimately recovered the preference for long-wavelength waves in fully nonlinear, two-dimensional simulations of the nighttime gradient drift instability. We can predict this result by revisiting the eikonal analysis.

We have used the electron density profile measured by Prakash et al. (1972) to perform an eikonal analysis representative of the nighttime electrojet. The results are shown in Fig. 6. Once again, the effects of anomalous electron mobility are taken into account. Intermediate- and largescale waves are considered individually in the top and bottom panels, respectively. We can see that the intermediate-scale waves continue to exhibit little dispersion. While it is true that the intermediate-scale waves generally have much larger growth rates than large-scale waves, the frequency contours shown in the top row of Fig. 6 are all open. Most of the intermediate-scale waves, therefore, are still transient in nature, even at night.

Note that, if an intermediate-scale wave were to emerge at an altitude of about $107 \mathrm{~km}$ where, in this case, $\nabla W$ becomes very small, and if it were to fall within the small region of $k_{z}-z$ space where the growth rate is positive, it would be absolutely unstable. Whether and where this situation actually occurs in nature depends on the shape of the background density and drift profiles. The emergence of an absolutely unstable intermediate-scale mode in a narrow band of altitudes seems to be a distinct possibility at night, however.

Consider next the large-scale dispersion curves plotted in the bottom row of Fig. 6. Despite the effects of anomalous electron mobility, finite density gradient scale length effects have reintroduced dispersion in this case and led to the reemergence of numerous closed frequency contours. Some of these fall in regions where the growth rate is positive; these correspond to unstable eigenmodes. Moreover, there are open contours which span altitudes from the bottom to the top of the electrojet region and which wind between the closed contours. These paths permit communication between the trapped regions. Waves launched near these contours will be able to migrate in altitude between the closed contours, growing in the unstable regions of $k_{z}-z$ space, tunnelling through the stable regions, and lingering near the closed regions. Such waves are convectively unstable. Note that the frequencies of the closed and nearly closed contours are small; the phase velocities of waves following these contours are apt to be quite small, and even wave packets following open contours can be long-lived.

The tunnelling feature of the large-scale waves was emphasized by $\mathrm{Hu}$ and Bhattacharjee (1999). Their numerical simulations of the nighttime gradient drift instability produced dominant kilometer-scale waves by the time dynamical equilibrium was achieved. These waves spanned altitudes that were alternately stable and unstable. Intermediate-scale waves continued to be present throughout the simulation and clustered in regions of the shear flow that were most favorable for growth. Both forward and inverse mode coupling were detected.

\section{Analysis}

Ronchi et al. (1991) described a non-steady, dynamical saturated equilibrium state in the daytime electrojet characterized by the dissipation of large-scale irregularities by shear and then diffusion, and a regeneration of the irregularities by nonlinear mode coupling from small to large scales. Their simulations were shown to be generally consistent with radar and sounding rocket observations, but the match was not definitive. Hu and Bhattacharjee (1998) presented simulation results predicting a saturated state characterized by isotropic, large-scale density irregularities that were also generally consistent with available observations. Discriminating between these theories requires information about the detailed morphology and dynamics of the electrojet flow.

Daytime in-beam imaging experiments support the theory and simulations presented by Ronchi et al. (1991). Their Fig. 16 virtually reproduces the images in the top row of Fig. 1, and their animated simulations are strikingly similar to animations made from the radar images. The predicted wavelength of the large-scale waves (about $2 \mathrm{~km}$ ), lifetime (about $30 \mathrm{~s}$ ), and predominance of constantly evolving, straining structures exhibiting a high degree of temporal variability all agree with observations. The predicted range of altitudes for wave formation, about $100-107 \mathrm{~km}$, is somewhat narrower than what is observed. This prediction is controlled by the linear dispersion relation, calculated on the basis of an electron density profile measured during project CONDOR 
(Pfaff et al., 1987). Better agreement should result from the use of a profile more representative of the actual conditions in question.

Nearly shear-free flows can also emerge in the equatorial E-region, as exemplified by the images in Fig. 2. Hysell and Burcham (2000) often observed very flat zonal phase velocity profiles in the daytime electrojet using interferometry. Recently, Hysell et al. (2002) showed that the lower thermospheric winds predicted by the National Center for Atmospheric Research General Circulation Model (NCAR TIMEGCM) tend to produce vertical polarization electric fields in the electrojet that partially negate and thereby flatten the background field profile. In the absence of strong shear, the equations describing gradient drift waves once again become normal, and the predominant waves should be predicted by the fastest growing local eigenmodes. The linear, local calculations performed by Ronchi et al. (1990), which retain the effects of turbulent electron mobility, predict that large-scale waves with wavelengths of about $500 \mathrm{~m}$ to $1 \mathrm{~km}$ should predominate in shear-free flows. This estimate agrees with the evidence of Fig. 2, although the range of altitudes predicted, again about 100 to $107 \mathrm{~km}$, is several kilometers higher than that observed.

Contrary to intuition, radar interferometer data show that large-scale waves in the electrojet continue to predominate at night, even though the density gradient scale lengths become very short. Hu and Bhattacharjee (1999) recovered this result using numerical simulations and attributed it to the ability of the large-scale waves to extend across stable regions of space and inhabit multiple unstable regions simultaneously. Using an eikonal analysis, we showed that the large-scale waves can exist as trapped or nearly trapped modes in the irregular nighttime ionosphere, while most of the faster growing intermediate-scale waves are transients. As this analysis predicted, the waves evident in Fig. 3 tend to extend over broad ranges of altitudes and undergo large excursions in altitude and orientation as they tunnel between regions of space that are locally unstable. Both the scattering intensity and the Doppler shift of the echoes observed are modulated as the waves propagate. Their zonal propagation speeds are uniformly small compared to the nominal electron drift speed.

Finally, Hu and Bhattacharjee (1999) predicted that strong, intermediate-scale waves should cluster in narrow altitude bands where the conditions for instability are most favorable. The eikonal analysis in Sect. 3 suggested that absolutely unstable, intermediate-scale modes could exist at night given appropriate background plasma density and drift profiles. The intense bands of diffuse scatter shown in Fig. 3 likely correspond to these fast-growing intermediate-scale modes. It may be possible to determine this from the ground if the spatial resolution of the imaging technique can be improved. Otherwise, in situ data are crucial for investigating the role of intermediate-scale waves in the nighttime electrojet.

\section{Discussion}

We have used a new imaging technique at Jicamarca to investigate in detail the structure and dynamics of large-scale waves in the equatorial electrojet and to evaluate numerical studies of the waves performed recently. The non-steady, shear-dominated dynamical equilibrium flow simulated by Ronchi et al. (1991) accurately predicts our daytime radar observations. That large-scale waves are observed to predominate at night, accompanied by intermediate-scale waves clustered in narrow altitude bands, was also predicted by the simulations described by $\mathrm{Hu}$ and Bhattacharjee (1999). The altitude extent of these large-scale waves, which can exceed $20 \mathrm{~km}$, was not explicitly predicted by those simulations, but is consistent with the linear, nonlocal theory presented here. These waves exist as trapped or nearly trapped wave modes that migrate between altitude regions that are locally unstable as they propagate slowly eastward. The waves are dispersive at night and are very coherent and long-lived. Neither shear nor nonlinear mode coupling influences the dynamics or morphology of the waves at night to the degree they do during the day.

While shear is generally present during the day and at night, the gradient length scale of the shear at night tends to be much longer than it is during the day, comparable to or longer than the wavelength of the large-scale waves, and much longer than typical nighttime density gradient scale lengths. Shear flow at night frequently exhibits amplitudes, phasing, and periods highly suggestive of neutral wind forcing (Hysell and Burcham, 2000). Consequently, large-scale waves are less influenced by shear at night than during the day. Moreover, since the large-scale waves that exist at night are trapped or nearly trapped modes inhabiting unstable regions of space much of the time, they do not depend upon shear-driven, nonlinear mode coupling for their continual regeneration as they do during the day. It is possible that the dynamics observed during the day could take place at night in regions of strong shear flow and relatively small background plasma density gradients. Some radar imaging data supports this hypothesis.

Our imaging experiments identified a new class of largescale primary waves that occur around 18:30LT. These waves emerge first at high altitudes, where the ions are marginally magnetized and then sweep continuously downward and westward into the altitude regime just above that inhabited by ordinary gradient drift waves. The phenomenon appears to be very common but has not been studied quantitatively. Such a study will require precise specification of the conductivity gradients, currents, winds, and electric fields in the vicinity of the solar terminator.

The analyzes presented here were intended to be suggestive of the types of wave phenomena that can arise in the daytime and nighttime electrojet. More specific, quantitative analyzes of in-beam radar images require concurrent information about the background plasma density and wind profiles and the background zonal electric field. In the past, such information has been obtainable only through sounding rocket 
experiments. Recently, Hysell et al. (2002) and Hysell and Chau (2001) introduced new techniques for measuring wind and density profiles in the electrojet using radar remote sensing. In the future, we hope to couple these new techniques with radar imaging, in order to analyze the dispersion of the large-scale waves as quantitatively as is possible.

\section{Appendix}

We derive here a linear model of the gradient drift instability in the equatorial electrojet, which takes into consideration height variations in the plasma density, mobilities, diffusivities, and polarization electric field. Simplified forms for the perpendicular electron and ion velocities are adopted as:

$$
\begin{aligned}
\mathbf{v}_{i} & =\frac{\Omega_{i}}{v_{\text {in }}} \frac{\boldsymbol{E}}{B}-\frac{C_{s i}^{2}}{v_{i n}} \nabla \ln n \\
& =\mu_{i} \boldsymbol{E}-D_{i} \nabla \ln n \\
\mathbf{v}_{e} & =\frac{\boldsymbol{E} \times \boldsymbol{B}}{B^{2}}-\frac{v_{e n}}{\Omega_{e}} \frac{\boldsymbol{E}}{B}-v_{e n} \frac{C_{s e}^{2}}{\Omega_{e}^{2}} \nabla \ln n \\
& =\mu_{H} \boldsymbol{E} \times \hat{b}-\mu_{e} \boldsymbol{E}-D_{e} \nabla \ln n,
\end{aligned}
$$

where $v_{\mathrm{jn}}, \Omega_{j}$, and $C_{s j}$ refer to the ion-neutral collision frequency, gyrofrequency, and sound speed for species $j$. Inserting these velocities into the continuity equations for fluid ions and electrons, the linearizing and assuming waves of the form $u(\mathbf{x}, t)=u(z) \exp \left(i\left(k_{x} x-\omega t\right)\right)$ yields the following system of one-dimensional equations for the perturbed density and electrostatic potential:

$$
\begin{aligned}
\left(\begin{array}{cc}
m_{11} & m_{12} \\
m_{21} & m_{22}
\end{array}\right)\left(\begin{array}{c}
n / n_{\circ} \\
\phi
\end{array}\right)=0 \\
m_{11}=-i \omega+2 \alpha n_{\circ}+i k_{x} \mu_{i} E_{x}+D_{i} k_{x}^{2}+\mu_{i} \frac{d E_{z}}{d z} \\
+\left(\mu_{i} E_{z}-\frac{d D_{i}}{d z}\right) \frac{d}{d z}-D_{i} \frac{d^{2}}{d z^{2}} \\
m_{12}=\mu_{i}\left(k_{x}^{2}-\frac{d^{2}}{d z^{2}}\right)-\frac{\mu_{i}}{L_{1}} \frac{d}{d z} \\
m_{21}=-i \omega+2 \alpha n_{\circ}-i k_{x}\left(\mu_{e} E_{x}+\frac{E_{z}}{B}\right)+D_{e} k_{x}^{2} \\
-\mu_{e} \frac{d E_{z}}{d z}-\left(\mu_{e} E_{z}+\frac{d D_{e}}{d z}-\frac{E_{z}}{B}\right) \frac{d}{d z} \\
-D_{e} \frac{d^{2}}{d z^{2}} \\
m_{22}=\mu_{e}\left(\frac{d^{2}}{d z^{2}}-k_{x}^{2}\right)+\frac{\mu_{e}}{L_{2}} \frac{d}{d z}-i k_{x} \frac{1}{L B} .
\end{aligned}
$$

Here, $E_{x}$ and $E_{z}$ are the background horizontal and vertical electric fields in the electrojet, $L$ is the vertical plasma density gradient scale length, and $L_{1}$ and $L_{2}$ are defined as:

$$
\begin{aligned}
L_{1}^{-1} & \equiv \frac{1}{n_{\circ} \mu_{i}} \frac{d}{d z} n_{\circ} \mu_{i} \\
L_{2}^{-1} & \equiv \frac{1}{n_{\circ} \mu_{e}} \frac{d}{d z} n_{\circ} \mu_{e} .
\end{aligned}
$$

Nontrivial solutions to the eigenvalue problem represented by (A1) for a given value of $k_{x}$ are the nonlocal eigenmodes with the eigenvalues $\omega$ and $\gamma$. We revert to the local solutions by assuming sinusoidal eigenfunctions. These are easily found algebraically and are plotted in the $k_{z}-z$ plane for given values of $k_{x}$ in Fig. 5 and Fig. 6.

Acknowledgements. This work was supported by the National Science Foundation through cooperative agreements ATM-9022717 and ATM-9408441 to Cornell University and by NSF grant ATM0080338 to Clemson University. The Jicamarca Radio Observatory is operated by the Geophysical Institute of Perú, Ministry of Education, with support from the NSF cooperative agreements just mentioned. The help of the staff, particularly R. F. Woodman, was much appreciated. This manuscript was prepared at the Radio Science Center for Space and Atmosphere at the Uji campus of Kyoto University. DLH is indebted to S. Fukao and M. Yamamoto for their advice, support, and hospitality.

Topical Editor M. Lester thanks C. Haldoupis and another referee for their help in evaluating this paper.

\section{References}

Ables, J. G.: Maximum entropy spectral analaysis, Astron. Astrophys. Suppl. Ser., 15, 383-393, 1974.

Bilitza, D., Rawer, K., Bossy, L., and Gulyaeva, T.: International Reference Ionosphere: - Past, present, future, Adv. Space Res., 13, \#3, 3-23, 1993.

Chapin, E. and Kudeki, E.: Plasma wave excitation on meteor trails in the equatorial electrojet, Geophys. Res. Lett., 21, 2433-2436, 1994.

Farley, D. T.: Theory of equatorial electrojet plasma waves: New developments and current status, J. Atmos. Terr. Phys., 47, 729744, 1985.

Farley, D. T., Ierkic, H. M., and Fejer, B. G.: Radar interferometry: A new technique for studying plasma turbulence in the ionosphere, J. Geophys. Res., 86, 1467-1472, 1981.

Farley, D. T., Swartz, W. E., Hysell, D. L., and Ronchi, C.: Highresolution radar observations of daytime kilometer-scale wave structure in the equatorial electrojet, J. Geophys. Res., 99, 299307, 1994.

Forbes, J. M.: The equatorial electrojet, Rev. Geophys., 19, 469504, 1981.

Fu, Z. F., Lee, L. C., and Huba, J. D.: A quasi-local theory of the $\boldsymbol{E} \times \boldsymbol{B}$ instability in the ionosphere, J. Geophys. Res., 91, 32633269, 1986.

Gagnepain, J.,Crochet, M., and Richmond, A. D.: Comparison of equatorial electrojet models, J. Atmos. Terr. Phys., 39, 1119$1124,1977$.

Haerendel, G. and Eccles, J. V.: The role of the equatorial electrojet in the evening ionosphere, J. Geophys. Res., 97, 1181-1192, 1992.

Haerendel, G., Eccles, J. V., and Cakir, S.: Theory for modeling the equatorial evening ionosphere and the origin of the shear in the horizontal plasma flow, J. Geophys. Res., 97, 1209-1223, 1992.

Hedin, A. E., et al.: Empirical wind model for the upper, middle, and lower atmosphere, J. Atmos. Terr. Phys., 58, 1421-1445, 1996.

Hoh, F. C.: Instability of Penning-type discharge, Phys. Fluids, 6, 1184-1191, 1963. 
$\mathrm{Hu}$, S. and Bhattacharjee, A.: Two-dimensional simulations of gradient-drift turbulence in the daytime equatorial electrojet, J. Geophys. Res., 103, 20 749-20 759, 1998.

$\mathrm{Hu}, \mathrm{S}$. and Bhattacharjee, A.: Gradient-drift instabilities and turbulence in the nighttime equatorial electrojet, J. Geophys. Res., 104, 28 123-28 132, 1999.

Huba, J. D. and Lee, L. C.: Short wavelength stabilization of the gradient drift instability due to velocity shear, Geophys. Res. Lett., 10, 357-360, 1983.

Hysell, D. L. and Burcham, J.: HF radar observations of quasiperiodic E-layer echoes over North America, J. Geophys. Res., 104, 4361-4371, 1999.

Hysell, D. L. and Burcham, J.: Ionospheric electric field estimates from radar observations of the equatorial electrojet, J. Geophys. Res., 105, 2443-2460, 2000.

Hysell, D. L. and Chau, J. L.: Inferring E-region electron density profiles at Jicamarca from coherent scatter, J. Geophys. Res., 106, 30 371-30 380, 2001.

Hysell, D. L. and Woodman, R. F.: Imaging coherent backscatter radar observations of topside equatorial spread F, Radio Sci., 32, 2309-2320, 1997.

Hysell, D. L., Chau, J. L., and Fesen, C. G.: Effects of large horizontal winds on the equatorial electrojet, J. Geophys. Res., in press, 2002.

Jaynes, E. T.: On the rationale of maximum-entropy methods, Proc. IEEE, 70, 939-952, 1982.

Kudeki, E. and Sürücü, F.: Radar interferometric imaging of fieldaligned plasma irregularities in the equatorial electrojet, Geophys. Res. Lett., 18, 41-44, 1991.

Kudeki, E., Farley, D. T., and Fejer, B. G.: Long wavelength irregularities in the equatorial electrojet, Geophys. Res. Lett., 9, 684-687, 1982.

Kudeki, E., Farley, D. T., and Fejer, B. G.: Theory of spectral asymmetries and nonlinear currents in the equatorial electrojet, J. Geophys. Res., 90, 429-436, 1985.

Kudeki, E., Fejer, B. G., Farley, D. T., and Hanuise, C.: The Condor equatorial electrojet campaign: Radar results, J. Geophys. Res., 92, 13 561-13 577, 1987.

Landau, L. D. and Lifshitz, E. M.: The Classical Theory of Fields, Butterworth-Heinmann, New York, 1997.

Mathews, J. D., Sulzer, M. P., and Perillat, P.: Aspects of layer electrodynamics inferred from high-resolution ISR observations of the 80-270 km ionosphere, Geophys. Res. Lett., 24, 14111414, 1997.

Pfaff, R. F.: Rocket observations of the equatorial electrojet: Current status and critical problems, J. Atmos. Terr. Phys., 53, 709728, 1991.

Pfaff, R. F., Kelley, M. C., Fejer, B. G., and Smiddy, M.: Rocket observations of coherent electrostatic waves on the topside of the auroral electrojet turbulent region (abstract), Eos Trans. AGU,
63, 393, 1982.

Pfaff, R. F., Kelley, M. C., Kudeki, E., Fejer, B. G., and Baker, K. D.: Electric field and plasma density measurements in the strongly driven daytime equatorial electrojet, 1, The unstable layer and gradient drift waves, J. Geophys. Res., 92, $13578-$ 13 596, 1987.

Prakash, S., Subbaraya, B. H., and Gupta, S. P.: Rocket measurements of ionization irregularities in the equatorial ionosphere at Thumba and identification of plasma irregularities, Indian J. Radio Space Phys., 1, 72-80, 1972.

Reddy, C. A. and Devasia, C. V.: Height and latitude structure of electric fields and currents due to local east-west winds in the equatorial electrojet, J. Geophys. Res., 86, 5751-5767, 1981.

Richmond, A. D.: Numerical model of the equatorial electrojet, Tech. Rep. AFCRL-72-0668, ERP 421, Air Force Cambridge Research Lab., Hanscom AFB, Bedford, MA, 1972.

Ronchi, C.: Large scale turbulence in the equatorial electrojet, Ph.D. thesis, Cornell Univ., Ithaca, New York, 1990.

Ronchi, C., Similon, P. L., and Sudan, R. N.: A nonlocal linear theory of the gradient drift instability in the equatorial electrojet, J. Geophys. Res., 94, 1317-1326, 1989.

Ronchi, C., Sudan, R. N., and Similon, P. L.: Effect of short-scale turbulence on kilometer wavelength irregularities in the equatorial electrojet, J. Geophys. Res., 95, 189-200, 1990.

Ronchi, C., Sudan, R. N., and Farley, D. T.: Numerical simulations of large-scale plasma turbulence in the daytime equatorial electrojet, J. Geophys. Res., 96, 21 263-21 279, 1991.

Simon, A.: Instability of a partially ionized plasma in crossed electric and magnetic fields, Phys. Fluids, 6, 382-388, 1963.

Skilling, J. and Bryan, R. K.: Maximum entropy image reconstruction: General algorithm, Mon. Not. R. Astron. Soc., 211, 111124, 1984.

St.-Maurice, J. P.: A unified theory of anomalous resistivity and Joule heating effects in the presence of ionospheric E-region irregularities, J. Geophys. Res., 92, 4533-4542, 1987.

Sudan, R. N., Unified theory of type I and type II irregularities in the equatorial electrojet, J. Geophys. Res., 88, 4853-4860, 1983.

Sudan, R. N., Akinrimisi, J., and Farley, D. T.: Generation of smallscale irregularities in the equatorial electrojet, J. Geophys. Res., 78, 240-248, 1973.

Swartz, W. E. and Farley, D. T.: High-resolution radar measurements of turbulent structure in the equatorial electrojet, J. Geophys. Res., 99, 309-317, 1994.

Thompson, A. R.: Interferometry and Synthesis in Radio Astronomy, John Wiley, New York, 1986.

Weinberg, S.: Eikonal method in magnetohydrodynamics, Phys. Rev., 126, 1899-1909, 1962.

Woodman, R. F.: Coherent radar imaging: Signal processing and statistical properties, Radio Sci., 32, 2373-2391, 1997. 